(C) The Author(s)

DOI: $10.1142 / \mathrm{S} 2010194518601096$

\title{
Recent developments in high-resolution-gamma-ray cargo-inspection technology
}

\author{
David Taylor \\ Department of Homeland Security, 1120 Vermont Avenue, NW \\ Washington, DC 20005, USA \\ david.taylor@HQ.DHS.GOV
}
Victor Orphan ${ }^{*}$, Eric Ackermann ${ }^{\dagger}$, Rhett Barnes ${ }^{\ddagger}$, Ryan Shyffer ${ }^{\S}$, George Mansfield ${ }^{\Uparrow}$ and James Winso"
Spectral Labs Incorporated, 15920 Bernardo Center Drive San Diego, CA 92127, USA
*orphanv@spectrallabs.com, ${ }^{*}$ ackermanne@spectrallabs.com, ${ }^{\star}$ barnesr@spectrallabs.com \$shyfferr@spectrallabs.com, "mansfieldg@spectrallabs.com,"winsoj@spectrallabs.com
James Christian $^{* *}$, Urmila Shirwadkar ${ }^{\dagger \dagger}$, Paul Bennett ${ }^{+\ddagger}$, Joshua Tower ${ }^{\S \S}$ and Kanai Shah"
Radiation Monitoring Devices, Inc., 44 Hunt Street, Watertown, MA 02472, USA
${ }^{* *}$ jchristian@rmdinc.com, ${ }^{\dagger \dagger}$ ushirwadkar@rmdinc.com, 㸵pbennett@rmdinc.com, ${ }^{\S}$ jtower@rmdinc.com, ${ }^{\top} k$ shah@rmdinc.com

Eduardo Padilla

Sandia National Laboratories, PO Box 5800 MS 1373

Albuquerque, NM 87185, USA

eapadil@sandia.gov

Published 10 July 2018

Under US Department of Homeland Security sponsorship, Spectral Labs Incorporated has developed a prototype high-resolution retrofit for an existing mobile VACIS, named the HighResolution Imaging System (HiRIS). The legacy $256 \mathrm{NaI}$ detectors in the VACIS detector column were replaced with $576 \mathrm{CsI}$ detectors, more than doubling the pixel count. Using SiPMs to replace conventional PMTs allowed the packing of more detectors in the same VACIS detector enclosure. Legacy analog signal-processing electronics were replaced with advanced digital signalprocessing electronics. Replacing gross counting in the legacy system with multichannel analysis of the counts from each detector will allow better control of detector crosstalk. The HiRIS detector modules were installed on a VACIS truck refurbished to as-new condition. Initial testing of the HiRIS prototype demonstrates enhanced spatial resolution by a factor of two as compared to the legacy system, without any degradation in throughput capability (20 containers per hour).

Keywords: Nonintrusive inspection; NII; cargo inspection; gamma imaging; SiPM.

PACS numbers: 07.85.-m, 87.59.-e, 42.79.Pw

This is an Open Access article published by World Scientific Publishing Company. It is distributed under the terms of the Creative Commons Attribution 4.0 (CC-BY) License. Further distribution of this work is permitted, provided the original work is properly cited. 


\section{Introduction}

The US Department of Homeland Security's Science and Technology Directorate is funding Spectral Labs Incorporated (SLI) to upgrade the performance and extend the useful life of mobile VACIS ${ }^{1}$ gamma-ray nonintrusive inspection (NII) systems used by US and other customs and border-protection agencies worldwide. This High-Resolution Imaging System (HiRIS) program will provide significant spatial-resolution enhancement of gamma cargo-inspection systems by doubling the pixel count. Along with improved contrast sensitivity and potentially greater penetration, the resulting system will enable quicker, more effective contraband interdiction by inspectors. This significant enhancement in spatial resolution is achieved by using the latest advances in photomultiplier technology and solid-state silicon photomultipliers (SiPM) coupled with higher-efficiency CsI scintillation crystals (replacing the larger NaI detectors used in legacy systems). The improved overall detection efficiency results in better contrast sensitivity while maintaining maximum scan speed and average throughput of 20 cargo conveyances per hour. SLI has developed digital pulse-shape analysis electronics and advanced image-processing algorithms to replace legacy analog electronics and analysis tools. These tools offer the potential to minimize crosstalk and provide sharper images. The HiRIS detector module and associated data processing, image-enhancement software, and processed-data display replace these functions in the legacy systems while maintaining the existing ${ }^{60} \mathrm{Co}$ source enclosure, collimator, and shutter, as well as the mechanical source, detector positioning system, and source/vehicle motion-control electronics. The HiRIS kit will also enable functioning at greater extremes of the operational environment required in many CBP locations.

\section{Detector Selection and Testing}

Three detector scintillation materials were considered: NaI, CsI, and BGO. Table 1 compares the properties and costs of these materials. We chose CsI based on stopping power, stability, noise floor, and cost.

Table 1. Comparison of properties of three candidate scintillation materials.

\begin{tabular}{|lccc|}
\hline & Nal:TI & CsI:TI & BGO \\
\hline Density & $3.67 \mathrm{~g} / \mathrm{cm}^{3}$ & 4.51 & 7.13 \\
\hline Zeff & 49.4 & 54.0 & 71.5 \\
\hline Hygroscopic & yes & slightly & no \\
\hline Attn. length @ 1.2 MeV & $5.24 \mathrm{~cm}$ & 4.32 & 2.44 \\
\hline PE (1.2 MeV) $2 \times 2 \times 7 \mathrm{~cm}^{3}$ & $4.2 \%$ & 6.7 & 24 \\
\hline Ttl Eff (1.2 MeV) $2 \times 2 \times 7 \mathrm{~cm}^{3}$ & $29 \%$ & 34 & 51 \\
\hline Emission & $415 \mathrm{~nm}$ & 550 & 480 \\
\hline Decay & $250 \mathrm{~ns}$ & 900 & 300 \\
\hline Light yield & $38,000 \mathrm{ph} / \mathrm{MeV}$ & 54,000 & 8,000 \\
\hline Est. SensL noise floor @ $70^{\circ} \mathrm{C}$ & $13 \mathrm{keV}$ & 36 & 112 \\
\hline Material cost (samples) & $\sim \$ 1752 \times 2 \times 7 \mathrm{~cm}^{3}$ & $\$ 1802 \times 2 \times 7 \mathrm{~cm}^{3}$ & $\$ 6502 \times 2 \times 7 \mathrm{~cm}^{3}$ \\
\hline
\end{tabular}

Based on cost, stopping power, noise floor and stability: Csl:Tl chosen 
Radiation Monitoring Devices, Inc. (RMD) supported SLI in designing packaging for the CsI, SiPM, and associated electronics. Figure 1 presents a preliminary detector package and several CsI crystals (with one face and all faces polished). Using this detector packaging, RMD tested over a dozen different combinations of CsI with four candidate SiPMs to evaluate noise performance after exposure to the range of projected operating temperatures, $-40^{\circ} \mathrm{C}$ to $70^{\circ} \mathrm{C}$. Figure 2 shows typical test results for an unsuccessful test at left and successful test at right. Note that the noise floor for the Ketex SiPM is $500 \mathrm{keV}$ at the $70^{\circ} \mathrm{C}$ temperature, whereas the SensL SiPM has an acceptable threshold noise of less than $200 \mathrm{keV}$ at the same temperature. RMD chose SensL and Hamamatsu SiPMs coupled with CsI crystals from two suppliers: Proteus and Epic. Use of two suppliers of SiPMs and CsI facilitated pricing competition.

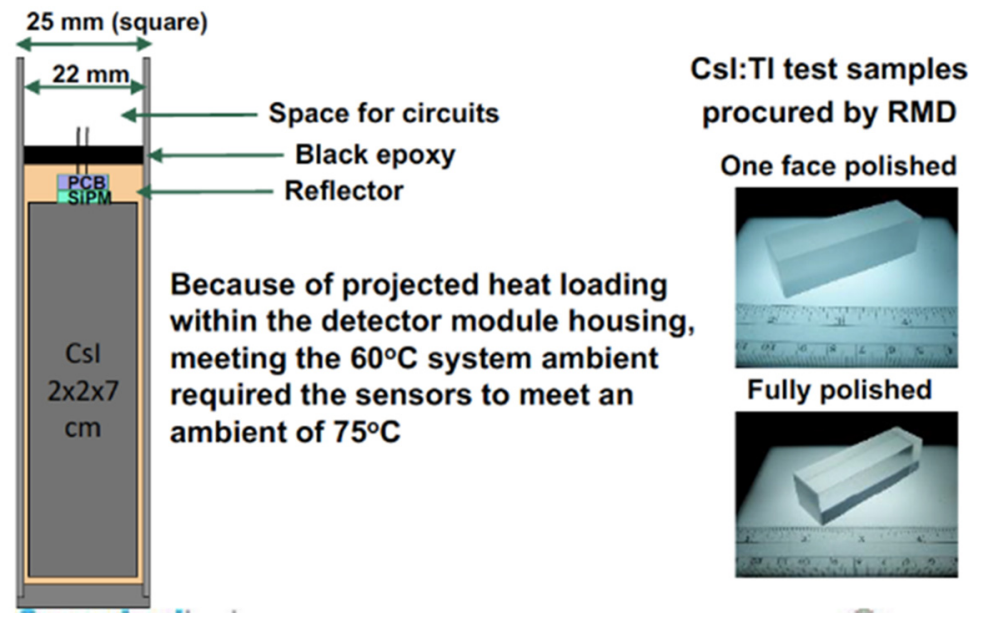

Fig. 1. Preliminary detector package and two CsI samples.

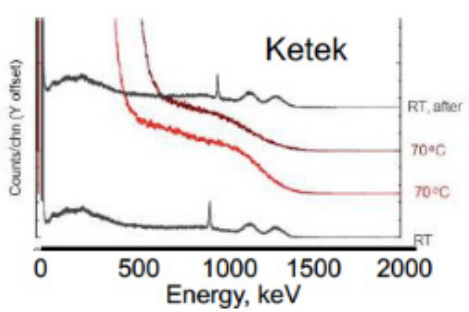

- Does not work at $70^{\circ} \mathrm{C}$, even after gain adjustment

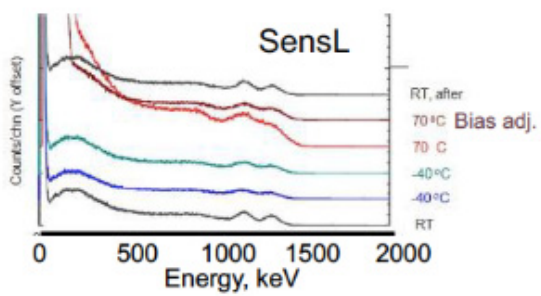

Noise threshold below $200 \mathrm{keV}$ AFTER adjusting operating bias

Fig. 2. Typical CsI-SiPM test results after temperature cycling. A tail-pulse generator produces the spike in the RT Ketek spectra at $\sim 850 \mathrm{keV}$. 


\section{Detector Modeling}

Sandia National Laboratories assisted our HiRIS detector development with MCNP modeling designed to investigate the effect of energy thresholds on crosstalk between detectors. HiRIS uses three columns of CsI detectors; for this reason, scattering from adjacent detectors may produce degradation (fuzziness) in image quality. The legacysystem detector featured gross counters with an energy threshold around $150 \mathrm{keV}$. Figure 3 illustrates how detector crosstalk can be minimized by dynamically changing the threshold from a low value for very dense cargo (where counting statistics are essential) to a higher threshold set around the ${ }^{60} \mathrm{Co}$ photopeaks for medium- and low-density cargo, resulting in improved image quality by mitigating detector crosstalk. The energy resolution of our CsI detectors is about $9 \%$ at $662 \mathrm{keV}$, which is adequate for HiRIS and helps reduce detector cost, since we can accept lower-quality CsI material.

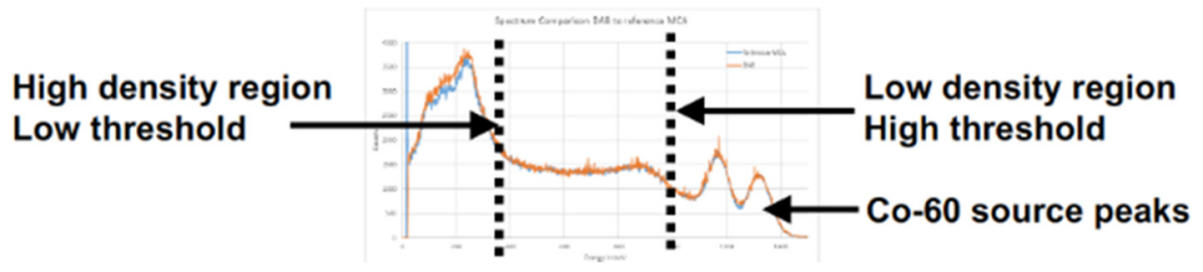

Fig. 3. Strategy for minimizing detector crosstalk by use of dynamic thresholds. Only high-energy counts are used in low-density cargo to effectively reduce crosstalk and sharpen the image.

Sandia used MCNP to model the response of a three-column array of CsI, using an inch-thick steel-plate target on which were placed a series of steel bars of different thicknesses. Setting the threshold around the photopeaks of the ${ }^{60} \mathrm{Co}$ source results in a much better definition of the edge of the density change in the target sample, as shown in Fig. 4. Lower thresholds allow detector-to-detector scatter, which degrades the sharpness of the density transition.

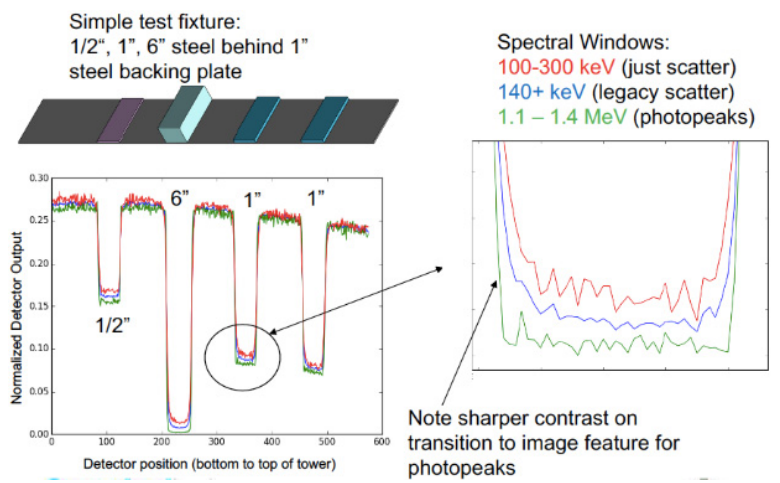

Fig. 4. Typical MCNP modeling results showing less detector-to-detector scattering for higher-energy threshold. 


\section{HiRIS Detector Integration into Gamma-Imaging Modules}

The strategy for packing more pixels in the same space uses smaller scintillators with solid-state SiPM to replace much-larger conventional PMTs. Figure 5 compares the sizes of the legacy NaI-PMT detector $\left(3.275 \mathrm{~cm}^{2}\right)$ and smaller CsI-SiPM detector $\left(2 \mathrm{~cm}^{2}\right)$. As shown in Fig. 5, the "form, fit, function" replacement of the legacy VACIS gamma-ray imaging detector involves swapping the old array tower (consisting of four modules, each with 64 detectors in two columns) for an arrangement of four modules, each with 144 detectors in three columns.

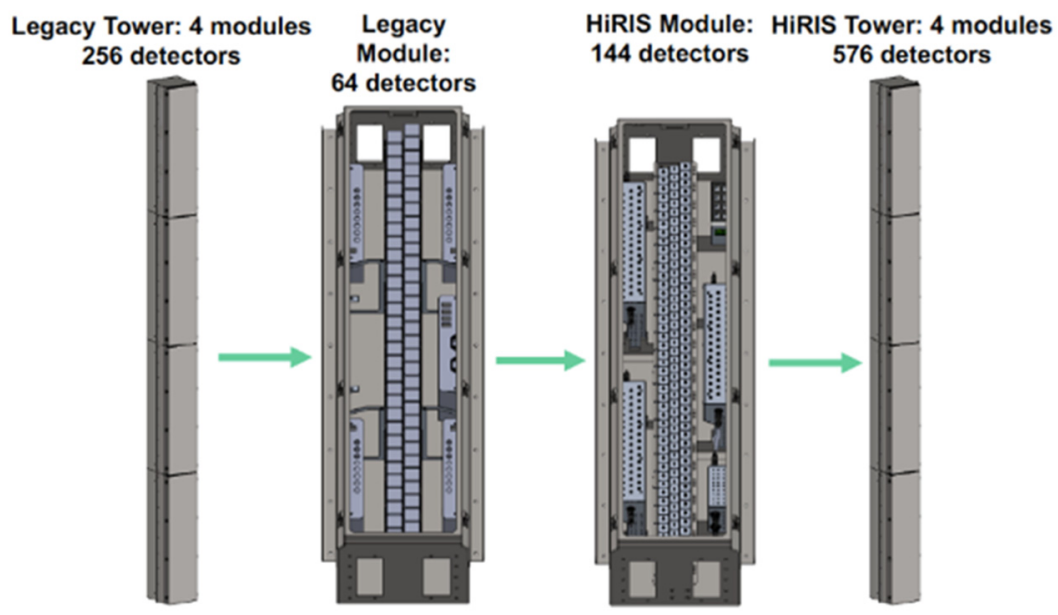

Fig. 5. HiRIS detector conversion overview.

\section{Integration of HiRIS Detector Modules with Vehicle and Preliminary Test Results}

Figure 6 shows the final assembly of $144 \mathrm{CsI}+\mathrm{SiPM}$ and associated electronics into a detector module. A VACIS legacy module with two columns of larger detectors is given for comparison.
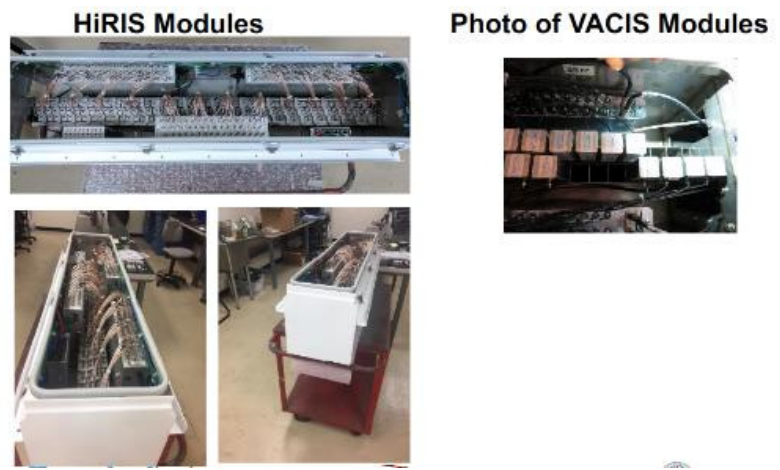

Fig. 6. Final assembly of HiRIS detector module, compared to VACIS detector module at right. 
The four HiRIS modules were tested at SLI and shipped to the AS\&E facility in Massachusetts for integration onto the refurbished mobile VACIS truck, as depicted in Fig. 7. Figure 8 shows the completion of full-scale integration and early testing of the HiRIS system. A preliminary scan of a manlift is also shown. Note that this gamma image does not include final digital-image processing and enhancement features, which are in development.

Check out of all four (4) modules after delivery to AS\&E before installation
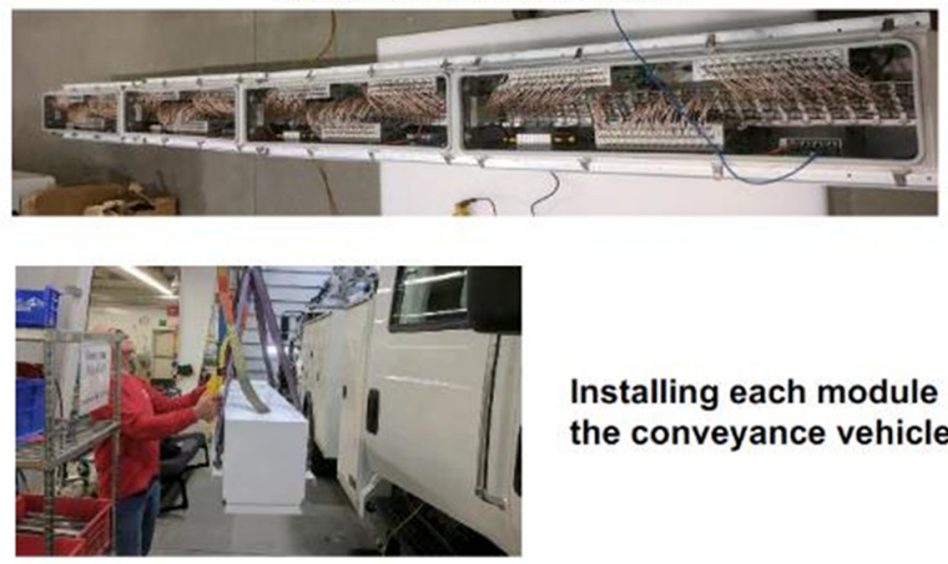

Installing each module on the conveyance vehicle

Fig. 7. Installation of the four HiRIS detector modules onto the VACIS truck.
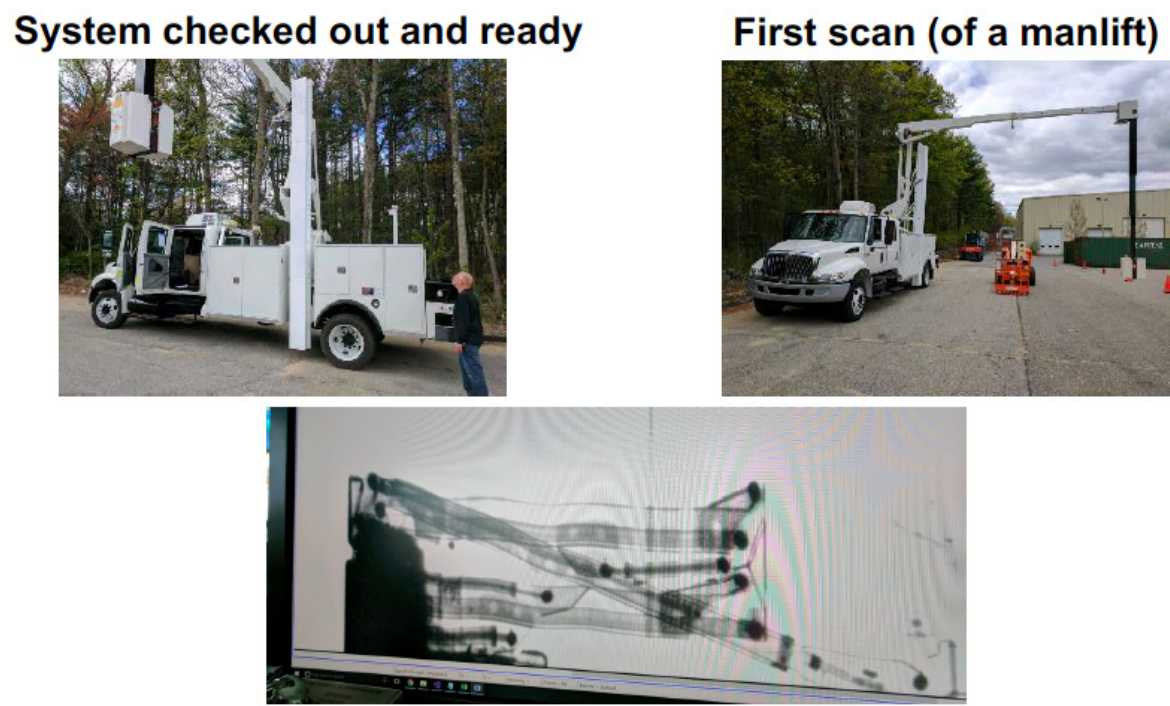

Early image (of manlift)

Fig. 8. Completion of HiRIS prototype and early image of manlift. 
Figure 9 shows the enhanced resolution of HiRIS (right) compared to that of the legacy VACIS system (left), using a standard resolution gauge consisting of steel wedges coming to a common point. Increasing the number of detectors (pixels) from 256 in the legacy system to 576 produces an improvement in spatial resolution by approximately a factor of two.

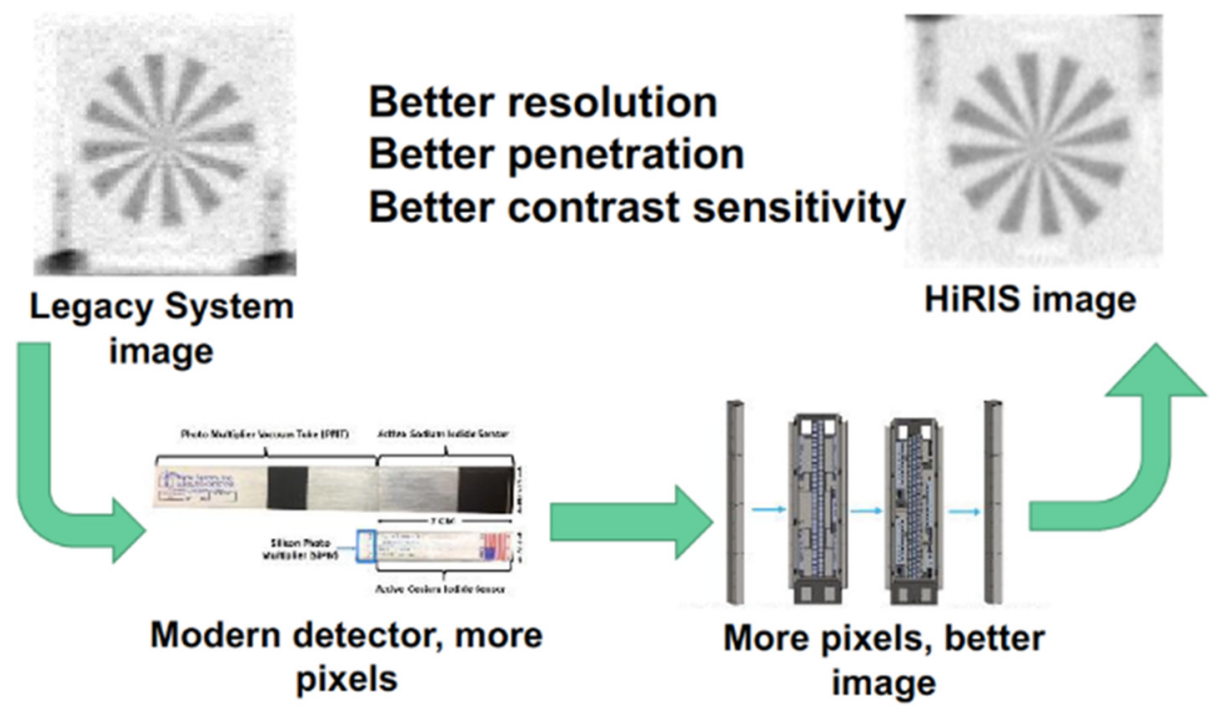

Fig. 9. Completion of HiRIS prototype and early image of manlift.

\section{Conclusion}

Once the HiRIS prototype has been successfully evaluated at a port of entry by Customs and Border Protection, the technology will afford a cost-effective means of enhancing the performance and lifespan of hundreds of VACIS systems. It will allow CBP and other VACIS users to avoid replacing aging VACIS systems with medium-energy X-ray systems that are less reliable, more costly, and require additional training for inspectors.

\section{Acknowledgments}

This project was funded by the Science and Technology Directorate of the United States Department of Homeland Security under contract number D15PC00264.

\section{Reference}

1. V. J. Orphan, VACIS Enhancements and Deployment Update, in Proceedings of the $7^{\text {th }}$ International Conference on Applications of Nuclear Techniques, Crete, Greece (June 2001). 